\title{
lodine Extravasation Quantification on Dual-Energy CT of the Brain Performed after Mechanical Thrombectomy for Acute Ischemic Stroke Can Predict Hemorrhagic Complications
}

\author{
(D) M. Bonatti, (D)F. Lombardo, (D) G.A. Zamboni, (DF. Vittadello, (D) R. Currò Dossi, D B. Bonetti, D R. Pozzi Mucelli, and (D) Gonatti
}

\begin{abstract}
BACKGROUND AND PURPOSE: Intracerebral hemorrhage represents a potentially severe complication of revascularization of acute ischemic stroke. The aim of our study was to assess the capability of iodine extravasation quantification on dual-energy CT performed immediately after mechanical thrombectomy to predict hemorrhagic complications.
\end{abstract}

MATERIALS AND METHODS: Because this was a retrospective study, the need for informed consent was waived. Eighty-five consecutive patients who underwent brain dual-energy CT immediately after mechanical thrombectomy for acute ischemic stroke between August 2013 and January 2017 were included. Two radiologists independently evaluated dual-energy CT images for the presence of parenchymal hyperdensity, iodine extravasation, and hemorrhage. Maximum iodine concentration was measured. Follow-up CT examinations performed until patient discharge were reviewed for intracerebral hemorrhage development. The correlation between dual-energy CT parameters and intracerebral hemorrhage development was analyzed by the Mann-Whitney $U$ test and Fisher exact test. Receiver operating characteristic curves were generated for continuous variables.

RESULTS: Thirteen of 85 patients (15.3\%) developed hemorrhage. On postoperative dual-energy CT, parenchymal hyperdensities and iodine extravasation were present in $100 \%$ of the patients who developed intracerebral hemorrhage and in $56.3 \%$ of the patients who did not $(P=.002$ for both). Signs of bleeding were present in $35.7 \%$ of the patients who developed intracerebral hemorrhage and in none of the patients who did not $(P<.001)$. Median maximum iodine concentration was $2.63 \mathrm{mg} / \mathrm{mL}$ in the patients who developed intracerebral hemorrhage and $1.4 \mathrm{mg} / \mathrm{mL}$ in the patients who did not $(P<.001)$. Maximum iodine concentration showed an area under the curve of 0.89 for identifying patients developing intracerebral hemorrhage.

CONCLUSIONS: The presence of parenchymal hyperdensity with a maximum iodine concentration of $>1.35 \mathrm{mg} / \mathrm{mL}$ may identify patients developing intracerebral hemorrhage with $100 \%$ sensitivity and $67.6 \%$ specificity.

ABBREVIATIONS: $\mathrm{DECT}=$ dual-energy $\mathrm{CT} ; \mathrm{ICH}=$ intracerebral hemorrhage; $\mathrm{PH}=$ parenchymal hematoma; $\mathrm{VNC}=$ virtual unenhanced reconstructions

M echanical thrombectomy in addition to intravenous tissue plasminogen activator administration has become the treatment of choice for patients with ischemic stroke secondary to a large-vessel occlusion. ${ }^{1,2}$ Despite the advances in patient selection, intracerebral hemorrhage ( $\mathrm{ICH}$ ) still represents a relevant complication of both mechanical thrombectomy and intravenous

Received July 21, 2017; accepted after revision November 5.

From the Departments of Radiology (M.B., F.L., G.B.) and Neurology (R.C.D., B.B.) Bolzano Central Hospital, Bolzano, Italy; Department of Radiology (G.A.Z., R.P.M.), University of Verona, Verona, Italy; and Explora-Research and Statistical Analysis (F.V.), Vigodarzere, Italy.

Preliminary data for this work were previously presented at: Annual Meeting of the European Congress of Radiology, March 1-5, 2017; Vienna, Austria.

Please address correspondence to Matteo Bonatti, MD, Department of Radiology, Bolzano Central Hospital, 5 Boehler St, 39100 Bolzano, Italy; e-mail:

matteobonatti@hotmail.com

http://dx.doi.org/10.3174/ajnr.A5513 thrombolysis, with a prevalence ranging from $4.3 \%$ to $43 \%$., ICHs following revascularization for acute ischemic stroke comprise hemorrhagic infarction and parenchymal hematoma (PH); hemorrhagic infarction is often asymptomatic, whereas $\mathrm{PH}$ is associated with high mortality rates and significantly worsened patient prognosis. ${ }^{5-7}$ Unenhanced CT is commonly performed within 24 hours after the treatment to rule out $\mathrm{ICH} .{ }^{8}$ Areas of increased attenuation can be observed in $33 \%-86 \%$ of the patients immediately after mechanical thrombectomy, mainly as a consequence of iodinated contrast material extravasation secondary to blood-brain barrier disruption, but the differential diagnosis with intracerebral hemorrhage can be challenging on singleenergy CT acquisitions. ${ }^{9-12}$

Dual-energy CT (DECT) has already proved to be helpful in differentiating contrast material extravasation and hemorrhage by subtracting the iodine component from the hyperdense area 
on virtual unenhanced reconstructions (VNC). ${ }^{9-11,13-16}$ However, late hemorrhagic complications may also occur in patients without bleeding signs on 24-hour CT. Given the impact of ICH on patient medical management (eg, platelet antiaggregants should be avoided in patients thought to have high bleeding risk), many algorithms have been developed to attempt to identify patients at higher risk for ICH development. ${ }^{4,5,17-21}$

The aim of our study was to assess the capability of iodine extravasation quantification on dual-energy CT performed immediately after mechanical thrombectomy to predict hemorrhagic complications.

\section{MATERIALS AND METHODS Patient Population}

This was a retrospective study approved by our institutional review board; the need for informed consent was waived. We considered for inclusion 114 consecutive patients who underwent mechanical thrombectomy at Bolzano Central Hospital between August 2013 and January 2017 because of acute ischemic stroke secondary to a large-vessel occlusion with a National Institutes of Health Stroke Scale score of $>6$ and symptom onset $<6$ hours for anterior circulation and $<12$ hours for posterior circulation. All patients underwent preprocedural unenhanced brain CT and CT angiography. Since 2007 in our institution, unenhanced brain CT is performed immediately after mechanical thrombectomy to rule out hemorrhagic complications; since August 2013, we acquire it with a dual-energy protocol to more confidently differentiate iodine extravasation from hemorrhage. ${ }^{14}$ The inclusion criterion was performance of unenhanced DECT of the brain within 1 hour after mechanical thrombectomy (91/114 patients). Exclusion criteria were unsatisfactory image quality because of motion artifacts (5/91 patients) and loss of DECT data (1/91 patients). Therefore, our study population included 85 patients, 52 men and 33 women, with a median age of 70 years (range, $31-87$ years). All patients underwent follow-up CT 24 hours after the procedure unless earlier due to clinical worsening; further follow-up CTs were performed according to clinical status.

\section{Imaging Protocols}

Endovascular procedures were performed on a biplane $\mathrm{x}$-ray system (Allura Xper FD20/20; Philips Healthcare, Best, the Netherlands) by 1 of a pool of 4 radiologists with 3-25 years of experience in interventional neuroradiology. All patients were treated according to a sequential approach that consisted of first-line aspiration using a $5 \mathrm{MAX} / 4 \mathrm{MAX}$ reperfusion catheter (Penumbra, Alameda, California) followed by mechanical thrombectomy with a Solitaire Flow-Restoration stent retriever (Covidien, Irvine, California) $)^{22-25}$ if aspiration was unsuccessful.

CT examinations were performed on a second-generation dual-source CT scanner (Somatom Definition Flash; Siemens, Erlangen, Germany) equipped with a tin filter. Immediate postoperative examinations were performed using a dual-energy technique $(80 / \mathrm{Sn} 140 \mathrm{kV}$ [peak]), whereas preoperative and follow-up examinations were performed using a single-energy technique $(120 \mathrm{kVp})$. All patients were positioned supine on the table with their arms along the body. Scanning parameters are reported in Table 1.
Table 1: Scanning parameters for postoperative unenhanced

dual-energy brain CT and follow-up unenhanced brain CT

\begin{tabular}{lll}
\hline & DECT & SECT \\
\hline Scanning technique & Spiral & Spiral \\
Scan direction & Caudocranial & Caudocranial \\
kVp & $80 / 140$ & 120 \\
mAs ref & $310 / 155$ & 390 \\
Collimation $(\mathrm{mm})$ & $40 \times 0.6$ & $128 \times 0.6$ \\
Rotation time $(\mathrm{sec})$ & 0.5 & 1 \\
Pitch & 0.7 & 0.55 \\
CARE Dose 4D $^{\mathrm{a}}$ & On & On \\
CARE-kV $^{\mathrm{a}}$ & Not available & Off \\
X-CARE & Not available & On \\
Kernel & D34f & H40s \\
\hline
\end{tabular}

Note:-SECT indicates single-energy CT; ref, reference.

a Siemens.

\section{Image Analysis}

Postoperative DECT images were independently and randomly evaluated by 2 radiologists with 8 and 5 years of experience in diagnostic neuroradiology, blinded to clinical data, using 3-mmthick multiplanar reconstructions. Window levels were set as suggested by the manufacturer and adjusted as needed. For each patient, simulated $120-\mathrm{kV}$ images, VNC images, and iodine map images were generated using commercially available software (syngo.via, Dual-Energy CT Brain Hemorrhage application, Version VA30A; Siemens). Simulated $120-\mathrm{kV}$ images represent a weighted average of the original low-kilovolt and high-kilovolt images, whereas VNC images and iodine map images are generated using a 3-material decomposition algorithm. For qualitative image analysis, the presence (yes/no), distribution (deep white matter, cortical-subcortical, or both), and homogeneity (homogeneous/inhomogeneous) of parenchymal hyperdensities were assessed on simulated $120-\mathrm{kV}$ images; the presence of iodine extravasation (yes/no) was assessed on iodine map images, and the presence of ICH (yes/no) was assessed on VNC images. Interreader discrepancies were solved by consensus. For quantitative image analysis, the average attenuation value (Hounsfield unit) at the site of maximum apparent attenuation (further reported as "maximum hyperdensity attenuation") was measured on simulated $120-\mathrm{kV}$ images by drawing a $0.3-\mathrm{cm}^{2}$ round ROI, and the average iodine concentration (milligrams/milliliter) at the site of maximum apparent iodine extravasation (further reported as "maximum iodine concentration") was measured on iodine map images by drawing a $0.3-\mathrm{cm}^{2}$ dual-energy ROI circle. The mean of the values obtained by the 2 readers was used for further analyses.

The Alberta Stroke Program Early CT Score was assessed on preoperative unenhanced $\mathrm{CT}$ of each patient by the 2 radiologists in consensus.

Finally, the 2 readers in consensus reviewed all the follow-up CTs, specifically looking for ICH development. ICH was defined by the presence of parenchymal hyperdensity persisting/increasing $>48$ hours after the procedure and/or by the appearance of new hyperdense lesions ${ }^{26}$; ICH on follow-up CTs was considered the reference standard for our study. ICHs were further subdivided according to the European Cooperative Acute Stroke Study classification ${ }^{3}$ into hemorrhagic infarction and parenchymal hematoma. 
Table 2: Results of postoperative DECT image analysis with interreader concordance and subsequent consensus

\begin{tabular}{|c|c|c|c|c|}
\hline & Reader 1 & Reader 2 & Interreader Concordance & Consensus \\
\hline \multicolumn{5}{|l|}{ Presence of parenchymal hyperdensity } \\
\hline Yes & $53 / 85(62.4 \%)$ & $53 / 85(62.4 \%)$ & $\kappa=0.95 ; 95 \% \mathrm{Cl}, 0.882-1.000$ & $54 / 85(63.5 \%)$ \\
\hline No & $32 / 85(37.6 \%)$ & $32 / 85(37.6 \%)$ & & $31 / 85(36.5 \%)$ \\
\hline \multicolumn{5}{|l|}{ Hyperdensity location } \\
\hline DWM & 26/53 (49.1\%) & $31 / 53(58.5 \%)$ & $\kappa=0.745 ; 95 \% \mathrm{Cl}, 0.588-0.903$ & $29 / 54(53.7 \%)$ \\
\hline DWM+cortical/subcortical & $21 / 53(39.6 \%)$ & $16 / 53(30.2 \%)$ & & $18 / 54(33.3 \%)$ \\
\hline Cortical/subcortical & $6 / 53(11.3 \%)$ & $6 / 53(11.3 \%)$ & & 7/54 (13.0\%) \\
\hline \multicolumn{5}{|l|}{ Hyperdensity homogeneity } \\
\hline Homogeneous & $29 / 53(54.7 \%)$ & $31 / 53(58.5 \%)$ & $\kappa=0.799 ; 95 \% \mathrm{Cl}, 0.632-0.966$ & $30 / 54(55.6 \%)$ \\
\hline Inhomogeneous & $24 / 53(45.3 \%)$ & $22 / 53(41.5 \%)$ & & $24 / 54(44.4 \%)$ \\
\hline Mean maximum attenuation (HU) & $\begin{array}{l}100 \text { (median } 77, \\
\text { range } 45-701)\end{array}$ & $\begin{array}{l}103 \text { (median } 76, \\
\quad \text { range } 47-789)\end{array}$ & $P=.999$ & 74 (range, 47-745) \\
\hline \multicolumn{5}{|l|}{ Presence of iodine extravasation } \\
\hline Yes & $53 / 85(62.4 \%)$ & $53 / 85(62.4 \%)$ & $\kappa=0.976 ; 95 \% \mathrm{Cl}, 0.929-1.000$ & $54 / 85(63.5 \%)$ \\
\hline No & $32 / 85(37.6 \%)$ & $32 / 85(37.6 \%)$ & & $31 / 85(36.5 \%)$ \\
\hline $\begin{array}{l}\text { Mean maximum iodine concentration } \\
\qquad(\mathrm{mg} / \mathrm{dL})\end{array}$ & $\begin{array}{l}3.0 \text { (median, } 1.8 ; \\
\quad \text { range } 0.5-28.4 \text { ) }\end{array}$ & $\begin{array}{l}2.9 \text { (median, 1.6; range } \\
0.6-27.7 \text { ) }\end{array}$ & $P=.926$ & 1.8 (range, $0.5-28.1)$ \\
\hline \multicolumn{5}{|l|}{ Hemorrhage on VNC images } \\
\hline Yes & $5 / 85(5.9 \%)$ & $5 / 85(5.9 \%)$ & $\kappa=1.000 ; 95 \% \mathrm{Cl}, 1.000-1.000$ & $5 / 85$ (5.9\%) \\
\hline No & $80 / 85(94.1 \%)$ & $80 / 85(94.1 \%)$ & & $80 / 85$ (94.1\%) \\
\hline
\end{tabular}

Note:-DWM indicates deep white matter.

\section{Clinical-Radiologic Data Collection}

A third physician, not involved in image analysis, retrieved, from our institutional data base, the following data for each patient: sex, age, time of symptom onset, National Institutes of Health Stroke Scale score at admission, site of vessel occlusion at preoperative CTA, preoperative intravenous tissue plasminogen activator administration (Actilyse; Boehringer Ingelheim, Ingelheim, Germany), general anesthesia during the interventional procedure, number of aspiration and thrombectomy attempts, arterial stent delivery, puncture-to-recanalization time, time to recanalization, and the postoperative Thrombolysis in Cerebral Infarction angiographic score.

\section{Statistical Analysis}

Continuous variables are reported as mean and/or median values and range (minimum and maximum); categoric variables, as numbers and percentages. Interobserver agreement was calculated according to weighted $\kappa$ statistics; agreement was considered very good if $\kappa=0.81-1.00$, good if $\kappa=0.61-0.80$, moderate if $\kappa=0.41-0.60$, fair if $\kappa=0.21-0.40$, and poor if $\kappa<0.20$. Interreader discrepancies for quantitative image analysis were evaluated using the Wilcoxon test for paired data. The comparison between subgroups ( $\mathrm{ICH}$ versus non-ICH) was performed with the Mann-Whitney $U$ test for continuous variables, whereas qualitative data were compared using the $\chi^{2}$ or Fisher exact tests (Fisher-Freeman-Halton test for $\mathrm{R} \times \mathrm{C}$ tables) when necessary. Receiver operating characteristic curves were calculated for continuous variables significantly associated with ICH. Multivariate logistic regression analyses (Wald statistic criteria, using the backward elimination method), adjusted for variables with a $P<.05$ on univariate analysis, were built to assess the value of these parameters to predict the risk of bleeding using a conditional forward procedure. Correlation between maximum iodine density and maximum attenuation was evaluated using the Spearman rank correlation coefficient. All tests were 2 -sided; $P$ values $<.05$ were considered statistically significant. Statistical analysis was performed using SPSS, Version 18.0 (IBM, Armonk, New York).

\section{RESULTS}

\section{Pre- and Perioperative Data}

Patients showed a mean NIHSS score of 20 (median, 20; range, 7-40) at admission. The mean ASPECTS at preoperative unenhanced brain CT was 8.9 (median, 10; range, 6-10). Seventy-six of $85(89.4 \%)$ patients had an anterior circulation stroke, whereas 9/85 (10.6\%) had a posterior circulation stroke. "Tandem" occlusion was present in 15/85 (17.6\%) cases. Tissue plasminogen activator was administered to $40 / 85(47.1 \%)$ patients. Fifty of 85 $(58.8 \%)$ procedures were performed with the patient under general anesthesia, and 35/85 (41.2\%), with the patient under conscious sedation. Fifty-eight of $85(68.2 \%)$ patients were treated with aspiration only, and 27/85 (31.8\%), with aspiration followed by stent-retriever thrombectomy. The median number of aspiration attempts was 2 (range, $0-8$ ). The median number of retrieval attempts in the $27 / 85$ patients who underwent stent-retriever thrombectomy was 2 (range, 1-4). A carotid artery stent was positioned in $6 / 85(7 \%)$ patients. The mean time to recanalization was 4 hours 43 minutes (range, 1 hour 25 minutes to 9 hours 40 minutes), and the mean puncture-to-recanalization time was 68 minutes (range, 15-167 minutes). The TICI score was 0 in 10/85 (11.8\%) patients; 1 in 5/85 (5.9\%); $2 \mathrm{a}$ in $15 / 85$ (17.6\%); $2 \mathrm{~b}$ in $16 / 85$ (18.8\%); and 3 in 39/85 (45.9\%); therefore, revascularization was considered successful in 55/85 (64.7\%) cases.

\section{Image Analysis}

The results of postoperative DECT image analysis with interreader concordance and subsequent consensus are reported in Table 2.

The 2 readers in consensus reviewed a median of 3 follow-up CTs per patient (range, 2-9). ICH was detected in 14/85 (16.5\%) patients: Six patients $(7.1 \%)$ showed hemorrhagic infarction, and 8 (9.4\%), PH. In 7/14 (50.0\%) patients, ICH was present 24 hours after the procedure; in $3 / 14(21.4 \%)$, it appeared $24-48$ hours after the procedure; in $2 / 14$ (14.3\%), 48-72 hours after the procedure; and in $2 / 14(14.3 \%),>72$ hours after the procedure. 

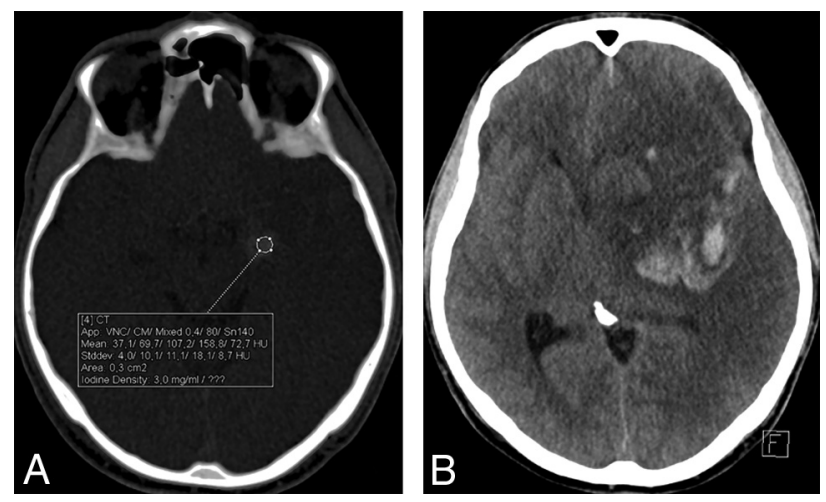

FIG 1. A, Postoperative DECT with an iodine map overlay shows a cortical-subcortical hyperdensity secondary to iodine extravasation with a maximum iodine concentration of $3.0 \mathrm{mg} / \mathrm{mL}$; no hyperdensity was visible on virtual unenhanced images (not included). B, A follow-up CT performed 48 hours later because of sudden clinical worsening shows a large parenchymal hematoma with a contralateral shift of the midline structures.
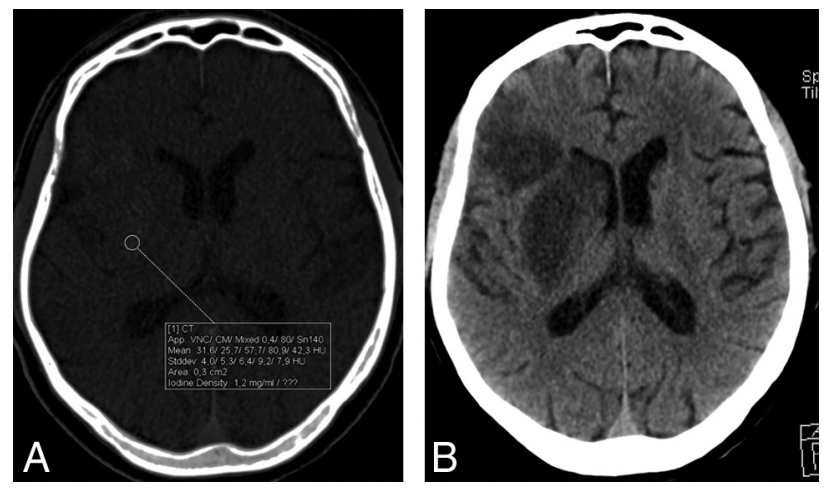

FIG 2. A, Postoperative DECT with an iodine map overlay shows a cortical-subcortical and deep white matter hyperdensity secondary to iodine extravasation with a maximum iodine concentration of 1.2 $\mathrm{mg} / \mathrm{mL}$; no hyperdensity was visible on virtual unenhanced images (not included). B, The follow-up CT performed before discharge, 4 days later, shows ischemic lesions at the sites of iodine extravasation but no hemorrhage.

\section{Imaging Findings: ICH Development Correlation}

On simulated $120-\mathrm{kV}$ images, parenchymal hyperdensity was present in 14/14 (100\%) patients who developed ICH and in $40 / 71(56.3 \%)$ patients who did not $(P=.002)$. Hyperdensity was inhomogeneous in 10/14 (71.4\%) patients who developed ICH and in $14 / 40(35.0 \%)$ patients who did not $(P=.028)$. Median maximum hyperdensity attenuation was $85 \mathrm{HU}$ (range, 63-745 $\mathrm{HU}$ ) in the group of patients who developed $\mathrm{ICH}$ and $68 \mathrm{HU}$ (range, 47-171 HU) in the group who did not $(P=.010)$. On iodine map images, iodine extravasation was present in $14 / 14$ (100\%) patients who developed ICH and in 40/71 (56.3\%) patients who did not $(P=.002)$. Median maximum iodine concentration was $2.63 \mathrm{mg} / \mathrm{mL}$ (range, $1.4-28.1 \mathrm{mg} / \mathrm{mL}$ ) in the group of patients who developed ICH and $1.40 \mathrm{mg} / \mathrm{mL}(0.5-4.2 \mathrm{mg} / \mathrm{mL})$ in the group who did not $(P<.001)$. A cutoff value of $1.35 \mathrm{mg} / \mathrm{mL}$ enabled identifying patients developing ICH with $100 \%$ sensitivity (95\% CI, $76.8 \%-100 \%)$ and $47.5 \%$ specificity (95\% CI, $31.5 \%-63.9 \%$ ), with a likelihood ratio of 1.905 (Figs 1 and 2).

On VNC images, signs of bleeding were present in 5/14 (35.7\%) patients who developed ICH. Complete data about the correlation between DECT findings and ICH development are reported in Table 3. Maximum hyperdensity attenuation showed an area under the curve of 0.73 (95\% CI, 0.60-0.87) for identifying patients developing ICH (Fig 3); a cutoff value of $67 \mathrm{HU}$ enabled identifying patients developing ICH with $92.9 \%$ sensitivity (95\% CI, 66.1\%-99.8\%) and $47.5 \%$ specificity (95\% CI, 31.5\%$63.9 \%$ ), with a likelihood ratio of 1.77 . Maximum iodine concentration showed an area under the curve of 0.89 (95\% CI, $0.68-$ 0.92 ) for identifying patients developing ICH (Fig 4); a cutoff value of $1.3 \mathrm{mg} / \mathrm{mL}$ enabled identifying patients developing ICH with $100 \%$ sensitivity (95\% CI, 76.8\%-100\%) and $47.5 \%$ specificity $(95 \%$ CI, $31.5 \%-63.9 \%)$, with a likelihood ratio of 1.90 . A significant correlation between maximum iodine concentration on iodine map images and maximum attenuation on simulated $120-\mathrm{kV}$ images was observed, with the Spearman $r=0.901$ (95\% CI, 0.832-0.943) $(P<.001)$. The 2 parameters showed a linear regression correlation with a slope of 20.27 (95\% CI, 18.3922.15) (Fig 5).

\section{Clinical Parameters: ICH Development Correlation}

At preoperative CT, the median ASPECTS was 8 in the patients who developed ICH and 10 in the patients who did not $(P=.008)$. ASPECTS showed an area under the curve of 0.71 (95\% CI, $0.57-$ 0.84 ) for identifying patients developing ICH; a cutoff value of 8 enabled identification of patients developing $\mathrm{ICH}$ with $57.1 \%$ sensitivity (95\% CI, $28.9 \%-82.3 \%)$ and $71.8 \%$ specificity $(95 \%$ CI, 59.9\%-81.9\%), with a likelihood ratio of 2.03 . Patient sex and age, NIHSS score at admission, site of vessel occlusion, tPA administration, general anesthesia, revascularization technique, number of aspiration attempts, number of stent retriever thrombectomy attempts, stent delivery, puncture-to-recanalization time, time to recanalization, and TICI score did not show any significant correlation with ICH development $(P>.05)$.

\section{Multivariate Analysis}

On multivariate analysis, it was impossible to elaborate a predictive model for the identification of patients developing $\mathrm{ICH}$. However, in our series, a diagnostic algorithm that subsequently evaluates the presence of parenchymal hyperdensity and maximum iodine concentration (Fig 6) enables identifying patients developing ICH with $100 \%$ sensitivity and $67.5 \%$ specificity.

\section{DISCUSSION}

The aim of our study was to assess the capability of iodine extravasation quantification on dual-energy CT performed immediately after mechanical thrombectomy to predict hemorrhagic complications.

Intracranial hemorrhage represents a well-known complication of acute ischemic stroke revascularization treatment, determining relevant changes in patient medical treatment and often worsening their outcome. In line with the data published in the literature, ${ }^{3,4,27}$ hemorrhagic complications were detected in $16.5 \%$ of the patients in our series; $7.1 \%$ of the patients showed a hemorrhagic infarction, which was asymptomatic in $83.3 \%$ of patients, whereas $9.4 \%$ developed a parenchymal hematoma, which always correlated with severe worsening of neurologic status and required decompressive craniectomy in $75 \%$ of the cases. 
Table 3: Univariate analysis-association between postoperative dual-energy CT parameters and subsequent intracerebral hemorrhage development ${ }^{a}$

\begin{tabular}{|c|c|c|c|}
\hline & $\begin{array}{c}\text { Non-ICH Group } \\
\begin{array}{c}(n=71) \\
\text { No. }(\%)\end{array}\end{array}$ & $\begin{array}{c}\text { ICH Group } \\
(n=14) \\
\text { No. (\%) }\end{array}$ & $P$ Value \\
\hline Hyperdensity on simulated 120-kV images & & & $.002^{\mathrm{b}}$ \\
\hline Yes & $40 / 71(56.3 \%)$ & 14/14 (100\%) & \\
\hline No & $31 / 71(43.7 \%)$ & $0 / 14(0 \%)$ & \\
\hline Hyperdensity site & & & .342 \\
\hline DWM & $23 / 40(57.5 \%)$ & $6 / 14(42.9 \%)$ & \\
\hline DWM + cortical & $11 / 40(27.5 \%)$ & $7 / 14(50.0 \%)$ & \\
\hline Cortical & $6 / 40(15.0 \%)$ & $1 / 14(7.1 \%)$ & \\
\hline Hyperdensity homogeneity & & & $.028^{\mathrm{b}}$ \\
\hline Homogeneous & $26 / 40(65.0 \%)$ & $4 / 14(28.6 \%)$ & \\
\hline Inhomogeneous & $14 / 40(35.0 \%)$ & $10 / 14(71.4 \%)$ & \\
\hline Hyperdensity maximum attenuation & & & $.010^{\mathrm{b}}$ \\
\hline Median (HU) & 68 & 85 & \\
\hline lodine extravasation on iodine map images & & & $.002^{\mathrm{b}}$ \\
\hline Yes & $40 / 71(56.3 \%)$ & 14/14 (100\%) & \\
\hline No & $31 / 71(43.7 \%)$ & $0 / 14(0 \%)$ & \\
\hline Maximum iodine concentration & & & $<.001^{\mathrm{b}}$ \\
\hline Median (mg/mL) & 1.40 & 2.63 & \\
\hline Hemorrhage on VNC images & & & $<.001^{\mathrm{b}}$ \\
\hline Yes & $0 / 71(0 \%)$ & 5/14 (35.7\%) & \\
\hline No & $71 / 71(100 \%)$ & $9 / 14(64.3 \%)$ & \\
\hline
\end{tabular}

Note:-DWM indicates deep white matter.

a The strength of the association is quantified by the $P$ value (significant if $P<.05$ ). b Significant.

$\mathrm{ROC}$ of Maximum attenuation $(\mathrm{HU})$

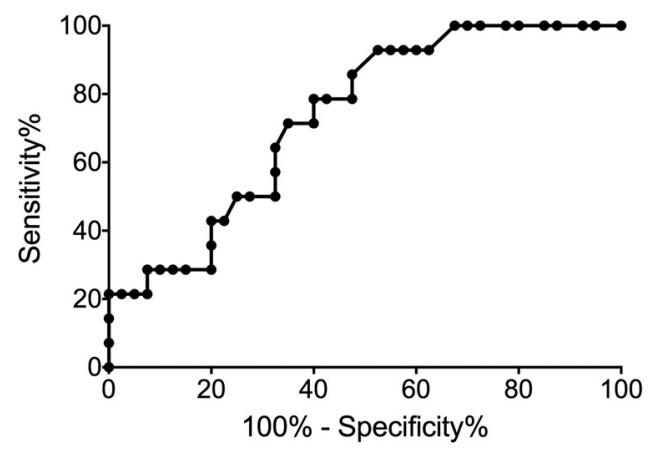

FIG 3. Receiver operator characteristic (ROC) curve showing sensitivity and specificity of maximum hyperdensity attenuation in identifying patients developing $\mathrm{ICH}$.

Although ICH typically appears within the first 24 hours after treatment, delayed bleeding may also occur; in our series, $50 \%$ of the ICHs appeared $>24$ hours after the procedure. The identification of patients with high bleeding risk is crucial to improving their clinical management and potential outcome; for example, antiaggregation should be delayed in these patients and arterial pressure should be strictly monitored to avoid hypertensive peaks. Therefore, many attempts have been made to identify patients at risk for ICH development, with variable results. ${ }^{5,17-19}$ In our series, ASPECTS $\leq 8$ at preoperative CT and the presence of parenchymal hyperdensity, hyperdensity inhomogeneity, hyperdensity attenuation of $>67 \mathrm{HU}$; iodine extravasation; maximum iodine concentration of $>1.35 \mathrm{mg} / \mathrm{mL}$; and bleeding signs on postoperative DECT were significantly correlated with $\mathrm{ICH}$ development. Our study also confirmed the reproducibility of DECT in the evaluation of patients after mechanical thrombec- tomy: All the evaluated parameters showed an interreader agreement ranging from "good" to "very good."

The integrity of the blood-brain barrier is governed by the tight junctions between endothelial cells, which regulate small molecule diffusion, and the basal lamina, a structural barrier preventing cellular extravasation. ${ }^{28}$ To our knowledge, only 1 article focused on the relationship between contrast extravasation and hemorrhage development. Yoon et $\mathrm{al}^{29}$ divided parenchymal hyperdensities after revascularization into contrast enhancement, defined as a hyperdense lesion without mass effect that disappeared on a 24-hour follow-up CT scan, and contrast extravasation, defined as a hyperdense lesion with a maximum measurement of $>90 \mathrm{HU}$ that persisted on a 24-hour follow-up CT scan. In their work, they found that the latter was associated with a higher rate of parenchymal hematomas; therefore, they postulated that contrast extravasation might be due to basal lamina disruption. Unlike the work of Yoon et al, our study was based on dual-energy CT acquisitions, which allowed a quantification of iodine extravasation; therefore, we focused on the possible relationship between iodine concentration and subsequent hemorrhage development. Moreover, the study by Yoon et al was based on patients who underwent intraarterial thrombolysis, whereas the patients included in our study underwent mechanical thrombectomy, a procedure that might incur some degree of endothelial damage.

In our series, there was a significant correlation between the maximum concentration of extravasated iodinated contrast material and subsequent hemorrhage development: Receiver operating characteristic analysis showed that the presence of hyperattenuating areas with maximum iodine concentration of $>1.35$ $\mathrm{mg} \mathrm{I} / \mathrm{mL}$ on postoperative DECT identified patients developing ICH with $100 \%$ sensitivity and $67.6 \%$ specificity. We chose the $1.35-\mathrm{mg} \mathrm{I} / \mathrm{mL}$ cutoff value because the main aim in postoperative patient management is to minimize hemorrhagic complications; therefore, a moderate specificity can be tolerated to obtain excellent sensitivity. According to our results, patients showing iodine extravasation with iodine concentration of $>1.35 \mathrm{mg} \mathrm{I} / \mathrm{mL}$ should not undergo postoperative antiaggregation/anticoagulation and should be more closely monitored to prevent hypertensive peaks.

We found a linear correlation between maximum iodine concentration and maximum hyperdensity attenuation, with a significant association between higher attenuation values and hemorrhage development, substantially confirming the results obtained by Yoon et al with single energy acquisitions. High attenuation may be a consequence of both bleeding and increased iodine extravasation, with the latter being a biomarker of blood-barrier damage, which predisposes to hemorrhage development. Simi- 
ROC of Maximum iodine concentration $(\mathrm{mg} / \mathrm{ml})$

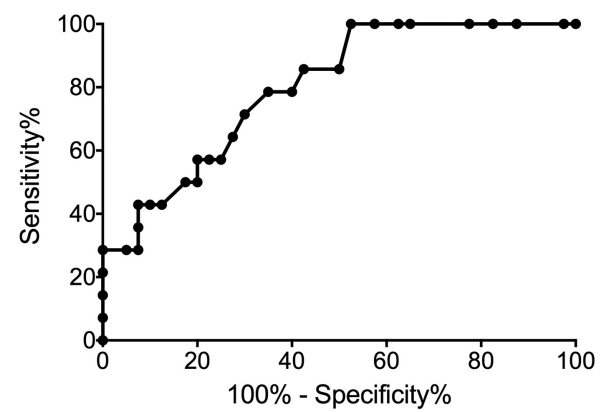

FIG 4. Receiver operator characteristic (ROC) curve showing sensitivity and specificity of maximum iodine concentration in identifying patients developing $\mathrm{ICH}$.

\section{Correlation iodine density/attenuation}

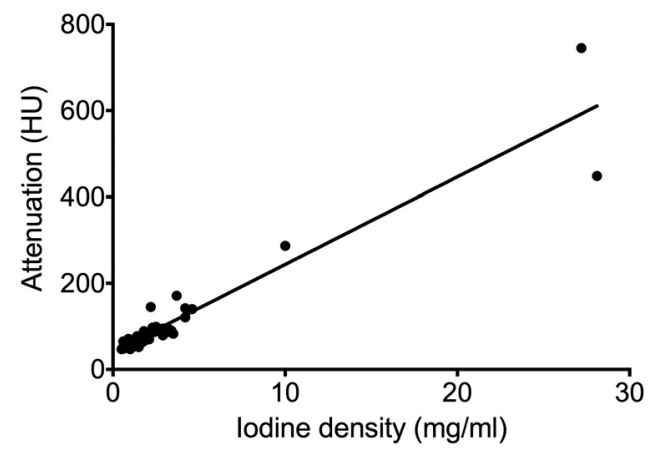

FIG 5. Graph showing the linear correlation existing between maximum hyperdensity attenuation and maximum iodine concentration.

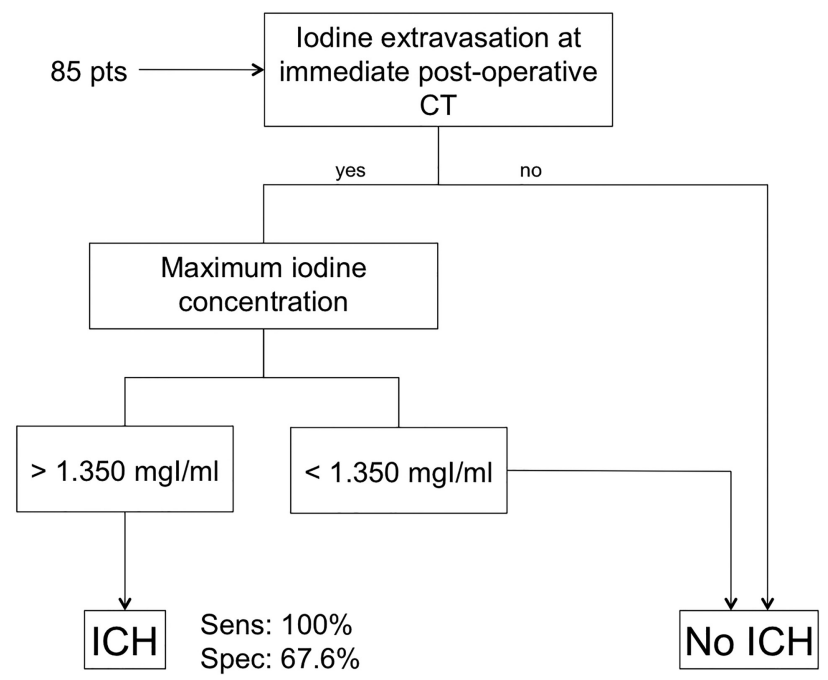

FIG 6. Diagnostic algorithm that enables identifying patents developing ICH with $100 \%$ sensitivity and $67.6 \%$ specificity.

larly, Nakano et al ${ }^{30}$ demonstrated that the absence of hyperdense areas at posttreatment CT represents a reliable negative predictor for symptomatic hemorrhage. In agreement with their results, none of the patients in our series without hyperattenuating areas on CT developed ICH.

In our series, we were not able to develop a predictive model to identify patients developing $\mathrm{ICH}$ on multivariate analysis, prob- ably because maximum iodine concentration was a strong predictor of subsequent hemorrhage development and no other factor helped in this prediction. Moreover, this failure might be a consequence of the low number of cases with positive findings (14/ 85); therefore, further studies with larger patient populations should be performed to confirm our findings.

Our study had several limitations, mainly correlated to its retrospective design. First, we used follow-up CT as the reference standard for identifying patients developing ICH because only a minority of the included patients underwent MR imaging. We are aware that some patients with no signs of bleeding on follow-up CT might have shown small hemosiderin deposits within the ischemic core at MR imaging, but we are also aware that the clinical relevance of these findings is at least questionable. Second, although all patients underwent a routine follow-up CT 24 hours after the procedure, further follow-up examinations were performed only according to clinical status. Therefore, it is possible that some patients who developed asymptomatic hemorrhagic infarction remained undiagnosed. However, these eventually missed hemorrhages were clinically irrelevant and did not affect patient outcome. Another limitation was that the 2 readers measured maximum iodine concentration at the site of maximum apparent iodine extravasation; therefore, the ROIs were subjectively drawn and might not reflect the real maximum iodine concentration in the affected area. However, we found high concordance in the measures performed by the 2 readers.

\section{CONCLUSIONS}

Iodine extravasation quantification on dual-energy CT performed immediately after mechanical thrombectomy for acute ischemic stroke may help identify patients with a higher risk of ICH development with excellent sensitivity and moderate specificity. This information might be useful for better tailoring the postoperative management to minimize hemorrhagic complications.

\section{REFERENCES}

1. Brinjikji W, Rabinstein A, Cloft HJ, et al. Recently published stroke trials: what the radiologist needs to know. Radiology 2015;276:8-11 CrossRef Medline

2. Yang P, Zhang Y, Liu J. LAST2 CH2ANCE: a summary of selection criteria for thrombectomy in acute ischemic stroke. AJNR Am J Neuroradiol 2017;38:E58-59 CrossRef Medline

3. Fiorelli M, Bastianello S, von Kummer R, et al. Hemorrhagic transformation within 36 hours of a cerebral infarct: relationships with early clinical deterioration and 3-month outcome in the European Cooperative Acute Stroke Study I (ECASS I) cohort. Stroke 1999;30: 2280-84 Medline

4. Khatri P, Wechsler LR, Broderick JP. Intracranial hemorrhage associated with revascularization therapies. Stroke 2007;38:431-40 CrossRef Medline

5. Paciaroni M, Agnelli G, Corea F, et al. Early hemorrhagic transformation of brain infarction: rate, predictive factors, and influence on clinical outcome: results of a prospective multicenter study. Stroke 2008;39:2249-56 CrossRef Medline

6. Wolpert SM, Bruckmann H, Greenlee R, et al. Neuroradiologic evaluation of patients with acute stroke treated with recombinant tissue plasminogen activator: the rt-PA Acute Stroke Study Group. AJNR Am J Neuroradiol 1993;14:3-13 Medline

7. Rao NM, Levine SR, Gornbein JA, et al. Defining clinically relevant cerebral hemorrhage after thrombolytic therapy for stroke: analysis of the National Institute of Neurological Disorders and Stroke 
tissue-type plasminogen activator trials. Stroke 2014;45:2728-33 CrossRef Medline

8. Higashida R, Furlan A, Roberts H, et al; Technology Assessment Committees of the American Society of Interventional and Therapeutic Neuroradiology and the Society of Interventional Radiology. Trial design and reporting standards for intraarterial cerebral thrombolysis for acute ischemic stroke. J Vasc Interv Radiol 2003;14(9 Pt 2): S493-94 Medline

9. Jang YM, Lee DH, Kim HS, et al. The fate of high-density lesions on the non-contrast CT obtained immediately after intra-arterial thrombolysis in ischemic stroke patients. Korean J Radiol 2006;7: 221-28 CrossRef Medline

10. Tijssen MP, Hofman PA, Stadler AA, et al. The role of dual energy CT in differentiating between brain haemorrhage and contrast medium after mechanical revascularisation in acute ischaemic stroke. Eur Radiol 2014;24:834-40 CrossRef Medline

11. Payabvash S, Qureshi MH, Khan SM, et al. Differentiating intraparenchymal hemorrhage from contrast extravasation on postprocedural noncontrast CT scan in acute ischemic stroke patients undergoing endovascular treatment. Neuroradiology 2014; 56:737-44 CrossRef Medline

12. Renú A, Amaro S, Laredo C, et al. Relevance of blood-brain barrier disruption after endovascular treatment of ischemic stroke: dual-energy computed tomographic study. Stroke 2015;46:673-79 CrossRef Medline

13. Kim JT, Heo SH, Cho BH, et al. Hyperdensity on non-contrast CT immediately after intra-arterial revascularization. J Neurol 2012; 259:936-43 CrossRef Medline

14. Gupta R, Phan CM, Leidecker C, et al. Evaluation of dual-energy CT for differentiating intracerebral hemorrhage from iodinated contrast material staining. Radiology 2010;257:205-11 CrossRef Medline

15. Phan CM, Yoo AJ, Hirsch JA, et al. Differentiation of hemorrhage from iodinated contrast in different intracranial compartments using dual-energy head CT. AJNR Am J Neuroradiol 2012;33:1088-94 CrossRef Medline

16. Bonatti M, Lombardo F, Zamboni GA, et al. Dual-energy CT of the brain: comparison between DECT angiography-derived virtual unenhanced images and true unenhanced images in the detection of intracranial haemorrhage. Eur Radiol 2017;27:2690-97 CrossRef Medline

17. Aviv RI, d'Esterre CD, Murphy BD, et al. Hemorrhagic transformation of ischemic stroke: prediction with CT perfusion. Radiology 2009;250:867-77 CrossRef Medline

18. Dorado L, Millan M, de la Ossa NP, et al. Influence of antiplatelet pre-treatment on the risk of intracranial haemorrhage in acute ischaemic stroke after intravenous thrombolysis. Eur J Neurol 2010; 17:301-06 CrossRef Medline
19. Sugiura Y, Yamagami H, Sakai N, et al; Committee of Recovery by Endovascular Salvage for Cerebral Ultra-acute Embolism (RESCUE)Japan Study Group. Predictors of symptomatic intracranial hemorrhage after endovascular therapy in acute ischemic stroke with large vessel occlusion. J Stroke Cerebrovasc Dis 2017;26:766-71 CrossRef Medline

20. Vandelli L, Marietta M, Gambini M, et al. Fibrinogen decrease after intravenous thrombolysis in ischemic stroke patients is a risk factor for intracerebral hemorrhage. J Stroke Cerebrovasc Dis 2015;24: 394-400 CrossRef Medline

21. Watson-Fargie T, Dai D, MacLeod MJ, et al. Comparison of predictive scores of symptomatic intracerebral haemorrhage after stroke thrombolysis in a single centre. J R Coll Physicians Edinb 2015;45: 127-32 CrossRef Medline

22. Lapergue B, Blanc R, Guedin P, et al. A Direct Aspiration, First Pass Technique (ADAPT) versus stent retrievers for acute stroke therapy: an observational comparative study. AJNR Am J Neuroradiol 2016;37:1860-65 CrossRef Medline

23. Massari F, Henninger N, Lozano JD, et al. ARTS (Aspiration-Retriever Technique for Stroke): initial clinical experience. Interv Neuroradiol 2016;22:325-32 CrossRef Medline

24. Romano DG, Cioni S, Leonini S, et al. Manual thromboaspiration technique as a first approach for endovascular stroke treatment: a singlecenter experience. Interv Neuroradiol 2016;22:529-34 CrossRef Medline

25. Romano DG, Cioni S, Vinci SL, et al. Thromboaspiration technique as first approach for endovascular treatment of acute ischemic stroke: initial experience at nine Italian stroke centers. J Neurointerv Surg 2017;9:6-10 CrossRef Medline

26. Khalilzadeh O, Sabel B, Sung Y, et al. Temporal evolution of intraparenchymal hyperdensity after intra-arterial therapy in patients with ischemic stroke: optimal discrimination between hemorrhage and iodinated contrast. Clin Neuroradiol 2014;24:365-71 CrossRef Medline

27. Elgendy IY, Kumbhani DJ, Mahmoud A, et al. Mechanical thrombectomy for acute ischemic stroke: a meta-analysis of randomized trials. J Am Coll Cardiol 2015;66:2498-505 CrossRef Medline

28. Hamann GF, Okada Y, del Zoppo GJ. Hemorrhagic transformation and microvascular integrity during focal cerebral ischemia/reperfusion. J Cereb Blood Flow Metab 1996;16:1373-78 CrossRef Medline

29. Yoon W, Seo JJ, Kim JK, et al. Contrast enhancement and contrast extravasation on computed tomography after intra-arterial thrombolysis in patients with acute ischemic stroke. Stroke 2004;35: 876-81 CrossRef Medline

30. Nakano S, Iseda T, Kawano H, et al. Parenchymal hyperdensity on computed tomography after intra-arterial reperfusion therapy for acute middle cerebral artery occlusion: incidence and clinical significance. Stroke 2001;32:2042-48 CrossRef Medline 